\title{
TWO HYPERBOLIC SCHWARZ LEMMAS
}

\section{Bernal-González and M.C. Calderón-Moreno}

In this paper, a sharp version of the Schwarz-Pick Lemma for hyperbolic derivatives is provided for holomorphic selfmappings on the unit disk with fixed multiplicity for the zero at the origin. This extends a recent result due to Beardon. A property of preserving hyperbolic distances also studied by Beardon is here completely characterised.

\section{INTRODUCTION AND NOTATION}

The Schwarz Lemma and its hyperbolic version, the Schwarz-Pick Lemma, continue to attract the attention of many mathematicians. Our aim in this paper is to prove two sharp versions of these results assuming that the multiplicity for the zero at the origin of the holomorphic function under consideration is fixed. Our results will extend a recent one due to Beardon, see below.

First of all, we need to fix some notation. The symbols $\mathbb{N}, \mathbb{C}, \mathbb{R}, \mathbb{D}, D(c, r)$ will denote, as usual, the set of positive integers, the complex plane, the real line, the open unit disk and the Euclidean closed disk $\{z \in \mathbb{C}:|z-c| \leqslant r\}(c \in \mathbb{C}, r>0)$, respectively. As for function spaces, $H(\mathbb{D})$ is the class of all holomorphic functions on $\mathbb{D}$ and Aut(D) will stand for the group of conformal automorphisms of $\mathbb{D}$. If $f \in H(\mathbb{D})$ and $a \in \mathbb{D}$ then $\mu(f, a)$ will represent the multiplicity for the zero at $a$ of the function $f(z)-f(a)$. For $m \in \mathbb{N}$ we introduce the notations

$$
\begin{aligned}
\mathcal{F}_{m} & =\left\{f \in H(\mathbb{D}):|f|<1, f(0)=f^{\prime}(0)=\cdots=f^{(m-1)}(0)=0\right\} \\
& =\{f \in H(\mathbb{D}):|f|<1, f(0)=0, \mu(f, 0) \geqslant m\}
\end{aligned}
$$

and

$$
z^{m} \operatorname{Aut}(\mathbb{D})=\left\{z^{m} f(z): f \in \operatorname{Aut}(\mathbb{D})\right\} .
$$

For the sake of convenience, we agree that $z^{0} \operatorname{Aut}(\mathbb{D})=\operatorname{Aut}(\mathbb{D})$. We say that a function $f$ is an $m$-rotation whenever there exists a constant $c$ with $|c|=1$ such that $f(z)=c z^{m}$. The set of all $m$-rotations will be represented by $\mathcal{R}_{m}$. It is clear that $z^{m-1} \operatorname{Aut}(\mathbb{D}) \cap \mathcal{F}_{m}=\mathcal{R}_{m}$. For $a \in \mathbb{D}$ we denote by $\varphi_{a}$ the special automorphism $\varphi_{a}(z)=((a-z) / 1-\bar{a} z)$. In fact,

$$
\operatorname{Aut}(\mathbb{D})=\left\{k \varphi_{a}:|a|<1=|k|\right\} .
$$

Received 3rd January, 2002

The authors have been partially supported by DGES Grant PB96-1348 and the Junta de Andalucía.

Copyright Clearance Centre, Inc. Serial-fee code: 0004-9727/02 \$A2.00+0.00. 
Note that $\varphi_{a}^{-1}=\varphi_{a}$. We define an m-automorphism of $\mathbb{D}$ as a function $f$ of the form $f=\psi \circ R \circ \varphi$ with $\varphi, \psi \in \operatorname{Aut}(\mathbb{D})$ and $R \in \mathcal{R}_{m}$ We denote by $\operatorname{Aut}_{m}(\mathbb{D})$ the set of $m$-automorphisms of $\mathbb{D}$. Obviously, $\operatorname{Aut}_{1}(\mathbb{D})=\operatorname{Aut}(\mathbb{D})$. It is straightforward to see that

$$
\operatorname{Aut}_{m}(\mathbb{D})=\left\{\varphi_{b} \circ R \circ \varphi_{a}: a, b \in \mathbb{D}, R \in \mathcal{R}_{m}\right\}
$$

and

$$
\left\{f \in \operatorname{Aut}_{m}(\mathbb{D}): f(a)=b\right\}=\left\{\varphi_{b} \circ R \circ \varphi_{a}: R \in \mathcal{R}_{m}\right\}
$$

The symbol $\rho$ will stand for the hyperbolic (or Poincaré) distance on $\mathbb{D}$, that is,

$$
\rho(z, w)=\tanh ^{-1}\left|\varphi_{z}(w)\right|=\frac{1}{2} \log \frac{1+|(z-w) /(1-\bar{z} w)|}{1-|(z-w) /(1-\bar{z} w)|} .
$$

If $f: \mathbb{D} \rightarrow \mathbb{D}$ is holomorphic then the hyperbolic derivative of order $m$ of $f$ at $z$ as introduced by Peschl is defined as

$$
f^{[m]}(z)=\left(\varphi_{f(z)} \circ f \circ \varphi_{z}\right)^{(m)}(0) .
$$

By using the fact $\varphi_{a}^{\prime}(t)=\left(|a|^{2}-1\right) /(1-\bar{a} t)^{2}$ together with Faa di Bruno's formula (see, for instance, $[\mathbf{3}])$ for the $m^{\text {th }}$ derivative of a composite function it is not difficult to check that

$$
f^{[1]}(z)=\frac{\left(1-|z|^{2}\right) f^{\prime}(z)}{1-|f(z)|^{2}}
$$

and that, for $m \geqslant 2$,

$$
f^{[m]}(z)=(-1)^{m+1} \frac{\left(1-|z|^{2}\right) f^{(m)}(z)}{1-|f(z)|^{2}}+\alpha(z),
$$

where $\alpha(z)$ is a finite sum of terms each of them containing at least one factor among $f^{\prime}(z), \ldots, f^{(m-1)}(z)$. Hence if $\mu(f, z) \geqslant m$ then $\alpha(z)=0$, so we have

$$
f^{[m]}(z)=(-1)^{m+1} \frac{\left(1-|z|^{2}\right) f^{(m)}(z)}{1-|f(z)|^{2}} .
$$

Hyperbolic derivatives are invariant in the sense that $\left|(S \circ f \circ T)^{[m]}\right|=\left|f^{[m]}\right| \circ T$ whenever $S$ and $T$ are conformal automorphisms of $\mathbb{D}$.

The Schwarz-Pick Lemma is a non-Euclidean version of the classical Schwarz Lemma. It asserts that

$$
\rho(f(z), f(w)) \leqslant \rho(z, w) \quad \text { and } \quad\left|f^{[1]}(z)\right| \leqslant 1
$$

for all $z, w \in \mathbb{D}$ and every $f \in H(\mathbb{D})$. Furthermore, the following are equivalent:

(i) $\rho(f(z), f(w))=\rho(z, w)$ for all $z$ and $w$ in $\mathbb{D}$ 
(ii) $\rho(f(z), f(w))=\rho(z, w)$ for some $z$ and $w$ in $\mathbb{D}$ such that $w \neq z$

(iii) $\left|f^{[1]}(z)\right|=1$ for all $z$ in $\mathbb{D}$

(iv) $\left|f^{[1]}(z)\right|=1$ for some $z$ in $\mathbb{D}$

(v) $f \in \operatorname{Aut}(\mathbb{D})$.

Recently, Beardon [1] has given an interesting new version of the Schwarz (or the Schwarz-Pick) Lemma. Specifically, he proved Theorem 1.1 below (see [1, Theorem] and notes following [1, Lemma 1]), which is a non-Euclidean version of a result due to Dieudonné [2] that establishes the following Schwarz Lemma for derivatives: If $f \in \mathcal{F}_{1}$ then

$$
\left|f^{\prime}(z)\right| \leqslant\left\{\begin{array}{lll}
1 & \text { if } & |z| \leqslant \sqrt{2}-1 \\
\frac{\left(1+|z|^{2}\right)^{2}}{4|z|\left(1-|z|^{2}\right)} & \text { if } & |z|>\sqrt{2}-1
\end{array}\right.
$$

This inequality is the best possible in terms of $|z|$. We now transcribe the statements of Beardon in our terminology (he denoted $f^{[1]}=f^{*}$ ). Recall that $f^{[1]}(z) \in \mathbb{D}$ if $f \notin \operatorname{Aut}(\mathbb{D})$. Beardon realised that this allows us to measure the hyperbolic distance between two hyperbolic derivatives.

ThEOREM 1.1. Assume that $f \in \mathcal{F}_{1} \backslash$ Aut $(\mathbb{D})$. We have:

(a) The inequality

$$
\rho\left(f^{[1]}(0), f^{[1]}(z)\right) \leqslant 2 \rho(0, z)
$$

is satisfied for all $z \in \mathbb{D}$.

(b) If equality holds in (1) for some $z \in \mathbb{D} \backslash\{0\}$ then $f \in z$ Aut(D)

(c) If $f(z)=z^{2}$ then equality holds in (1) for all $z \in \mathbb{D}$.

\section{A PRELIMINARY RESULT}

Before stating our theorems, we need an elementary lemma which is an " $m$-order" generalisation of the Schwarz-Pick Lemma.

Lemma 2.1. Assume that $m \in \mathbb{N}, a \in \mathbb{D}, f \in H(\mathbb{D}),|f|<1$ on $\mathbb{D}$ and $\mu(f, a)$ $\geqslant m$. Then we have

$$
\left|\frac{f(z)-f(a)}{1-\overline{f(a)} f(z)}\right| \leqslant\left|\frac{z-a}{1-\bar{a} z}\right|^{m} \quad(z \in \mathbb{D})
$$

and $\left|f^{[m]}(a)\right| \leqslant m !$

Further, equality holds in (2) for all $z \in \mathbb{D}$ if and only if it holds for some $z \neq a$ if and only if $\left|f^{[m]}(a)\right|=m$ ! if and only if $f \in \operatorname{Aut}_{m}(\mathbb{D})$. 
Proof: If $a \in \mathbb{D}$ satisfies $\mu(f, a) \geqslant m$ then the function

$$
F(t)=\frac{\left(\varphi_{b} \circ f \circ \varphi_{a}\right)(t)}{t^{m}} \quad(t \in \mathbb{D} \backslash\{0\})
$$

has a holomorphic extension to $\mathbb{D}$ because $\mu\left(\varphi_{b} \circ f \circ \varphi_{a}, 0\right)=\mu(f, a) \geqslant m$, where we have denoted $b=f(a)$. Fix $r \in(0,1)$. Then $|F(t)| \leqslant 1 / r^{m}$ on $|t|=r$, hence an application of the Maximum Modulus Principle yields $\sup \{|F(t)|:|t| \leqslant r\} \leqslant 1 / r^{m}$. Letting $r \rightarrow 1$ we get $\sup \{|F(t)|:|t|<1\} \leqslant 1$, that is, $|F(t)| \leqslant 1$ on $\mathbb{D}$ or, equivalently, $\left|\varphi_{b} \circ f \circ \varphi_{a}(t)\right| \leqslant|t|^{m}(t \in \mathbb{D})$, which becomes (2) after the change of variable $z=\varphi_{a}(t)$. Note that the value of (the extension of) $F$ at the origin is

$$
\begin{aligned}
F(0) & =\lim _{t \rightarrow 0} \frac{1}{t^{m}} \cdot \frac{b-f\left(\varphi_{a}(t)\right)}{1-\bar{b} f\left(\varphi_{a}(t)\right)} \\
& =\lim _{z \rightarrow a} \frac{(1-\bar{a} z)^{m}}{1-\overline{f(a)} f(z)} \cdot \frac{f(z)-f(a)}{(z-a)^{m}} \cdot(-1)^{m+1} \\
& =(-1)^{m+1} \cdot \frac{\left(1-|a|^{2}\right)^{m}}{1-|f(a)|^{2}} \cdot \lim _{z \rightarrow a} \frac{f(z)-f(a)}{(z-a)^{m}} \\
& =\frac{f^{[m]}(a)}{m !}
\end{aligned}
$$

after using the L'Hôpital's rule together with the definition of the hyperbolic derivative of order $m$ and the fact that $f^{\prime}(a)=\cdots=f^{(m-1)}(a)=0$. Again by the Maximum Modulus Principle, $|F(0)| \leqslant 1$, whence $\left|f^{[m]}(a)\right| \leqslant m !$.

Assume now that $f$ is an $m$-automorphism. Since $f(a)=b$ we have $f=\varphi_{b} \circ R \circ \varphi_{a}$ where $R \in \mathcal{R}_{m}$, that is, $R(t)=c t^{m}$ for some $c$ with $|c|=1$. Therefore $F(t)=c$ on $\mathbb{D}$, so $|F|=1$ on $\mathbb{D}$ and the equality holds in (2) for all $z \in \mathbb{D}$ (hence for some $z \neq a$ ). Moreover,

$$
\left|f^{[m]}(a)\right|=m ! \cdot|F(0)|=m ! \cdot|c|=m ! .
$$

Conversely, let $\left|f^{[m]}(a)\right|=m$ !. Then $|F(0)|=1$ and the Maximum Modulus Principle tells us that $F(t)=c$ for some unimodular constant $c$, but this yields $\left(\varphi_{b} \circ f \circ \varphi_{a}\right)(t)=c t^{m}$ for all $t \in \mathbb{D}$, which in turn implies that $f=\varphi_{b} \circ R \circ \varphi_{a}$ with $R$ as before. Consequently, $f$ is an $m$-automorphism of $\mathbb{D}$.

Finally, assume that equality holds in (2) for some $z \neq a$. Then the change $z=\varphi_{a}(t)$ shows that $\left|\varphi_{b} \circ f \circ \varphi_{a}(t)\right|=|t|^{m}$ for some $t \neq 0$, whence $|F(t)|=1$ for some $t \in \mathbb{D}$. Another application of the Maximum Modulus Principle drives us to $F(t)=c$ on $\mathbb{D}$ for some unimodular constant $c$, and this implies as above that $f \in \operatorname{Aut}_{m}(\mathbb{D})$. This concludes the proof.

\section{MAIN RESUltS}

We are now ready to state our theorems. As in [1], we can estimate the hyperbolic distance between two normalised hyperbolic derivatives of higher order under obvious 
conditions.

ThEOREM 3.1. Assume that $f \in \mathcal{F}_{m} \backslash \operatorname{Aut}_{m}(\mathbb{D})$. We have:

(a) If $a \in \mathbb{D}$ and $\mu(f, a) \geqslant m$ then

$$
\rho\left((-1)^{m+1} \frac{f^{[m]}(0)}{m !}, \frac{f^{[m]}(a)}{m !}\right) \leqslant 2 \rho(0, a) .
$$

(b) If there exists $a \in \mathbb{D} \backslash\{0\}$ for which $\mu(f, a) \geqslant m$ such that equality holds in (3) then $f \in z^{m}$ Aut $(\mathbb{D})$.

Proof: Observe that if $\mu(f, a) \geqslant m$ and $f \in \mathcal{F}_{m} \backslash$ Aut $_{m}(\mathbb{D})$ then the values $(-1)^{m+1}\left(f^{[m]}(0)\right) / m !$ and $\left(f^{[m]}(a)\right) / m$ ! are in $\mathbb{D}$ by Lemma 2.1 , so the hyperbolic distance between them makes sense. As for (a), since $\mu(f, a) \geqslant m \leqslant \mu(f, 0)$ the functions

$$
g(z)=\frac{f(z)}{z^{m}} \quad(z \in \mathbb{D} \backslash\{0\})
$$

and

$$
h(z)=\left(\frac{1-\bar{a} z}{a-z}\right)^{m} \cdot \frac{f(a)-f(z)}{1-\overline{f(a)} f(z)} \quad(z \in \mathbb{D} \backslash\{a\})
$$

have holomorphic extensions on the whole of $\mathbb{D}$ if we set

$$
g(0)=\frac{f^{(m)}(0)}{m !}=(-1)^{m+1} \frac{f^{[m]}(0)}{m !}
$$

and

$$
h(a)=\lim _{z \rightarrow a} h(z)=\frac{\left(1-|a|^{2}\right)^{m}}{1-|f(a)|^{2}} \cdot(-1)^{m+1} \cdot \frac{f^{(m)}(a)}{m !}=\frac{f^{[m]}(a)}{m !} .
$$

We may start with $a \neq 0$, since the case $a=0$ is trivial.

Note that by Lemma 2.1 (as applied on points $0, a$ ) we get $|g| \leqslant 1,|h| \leqslant 1$ on $\mathbb{D}$, and in fact $|g|<1,|h|<1$ on $\mathbb{D}$ since $f$ is not an $m$-automorphism. On the other hand, $g(a)=\left(f(a) / a^{m}\right)$ and $h(0)=\left(f(a) / a^{m}\right)$. If we apply the Schwarz-Pick Lemma to $g$ and $h$ then one obtains $\rho(g(0), g(a)) \leqslant \rho(0, a)$ and $\rho(h(0), h(a)) \leqslant \rho(0, a)$. Consequently, observing that $g(a)=h(0)$, the triangle inequality yields

$$
\rho\left((-1)^{m+1} \frac{f^{[m]}(0)}{m !}, \frac{f^{[m]}(a)}{m !}\right)=\rho(g(0), h(a)) \leqslant \rho(0, a)+\rho(0, a)=2 \rho(0, a),
$$

which proves (a). In order to prove (b), assume that equality in (3) holds for some $a \in \mathbb{D} \backslash\{0\}$ with $\mu(f, a) \geqslant m$. Then

$$
2 \rho(0, a)=\rho(g(0), h(a)) \leqslant \rho(g(0), g(a))+\rho(h(0), h(a)) \leqslant 2 \rho(0, a),
$$

whence $\rho(g(0), g(a))=\rho(0, a)$ because both terms in the last sum are not greater than $\rho(0, a)$. But the Schwarz-Pick Lemma tells that $g \in \operatorname{Aut}(\mathbb{D})$, hence $f \in z^{m} \operatorname{Aut}(\mathbb{D})$. The proof is finished. 
Corollary 3.2. If $m$ is even and $f \in \mathcal{F}_{m} \backslash \operatorname{Aut}_{m}(\mathbb{D})$ then $f^{(m)}(0)=0$. In other words, $f \in \mathcal{F}_{m+1}$.

Proof: From (3) and the fact that $\mu(f, 0) \geqslant m$ we obtain

$$
\rho\left(\frac{f^{[m]}(0)}{m !},-\frac{f^{[m]}(0)}{m !}\right) \leqslant 2 \rho(0,0)=0
$$

therefore $f^{[m]}(0)=-f^{[m]}(0)$. Consequently,

$$
f^{(m)}(0)=(-1)^{m+1} f^{[m]}(0)=0 .
$$

It should be noted that parts (a) and (b) of Theorem 1.1 are covered by the case $m=1$ in Theorem 3.1 (observe that always $\mu(f, a) \geqslant 1$ ). An extension of part (c) of Theorem 1.1 makes no sense because if $m \geqslant 2$ then the set $\{z \in \mathbb{D}: \mu(f, z) \geqslant m\}$ is discrete in $\mathbb{D}$ except for the trivial case $f \equiv 0$. In view of parts (b) and (c) of Theorem 1.1 one can wonder whether $f \in z \operatorname{Aut}(\mathbb{D})$ implies equality in (1) for some (or even for all) $z \in \mathbb{D} \backslash\{0\}$. In fact, we have been able to discover the exact conditions under which equality holds in (1). This will be accomplished in the following theorem, which strengthens Beardon's result.

ThEOREM 3.3. Let $f \in \mathcal{F}_{1} \backslash \operatorname{Aut}(\mathbb{D})$. We have:

(a) The inequality

$$
\rho\left(f^{[1]}(0), f^{[1]}(z)\right) \leqslant 2 \rho(0, z)
$$

is satisfied for all $z \in \mathbb{D}$.

(b) The equality

$$
\rho\left(f^{[1]}(0), f^{[1]}(z)\right)=2 \rho(0, z)
$$

holds for some $z \in \mathbb{D} \backslash\{0\}$ if and only if it holds for all points of a diameter of $\mathbb{D}$ if and only if $f \in z \operatorname{Aut}(\mathbb{D})$.

(c) The above equality holds for all $z \in \mathbb{D} \backslash\{0\}$ if and only if it holds for two nonzero points lying in two distinct diameters of $\mathbb{D}$ if and only if $f$ is a 2-rotation.

Proof: Part (a) is as in Theorem 1.1. It has been transcribed for the sake of completeness. As for (b) and (c), if equality (4) holds for some $z \in \mathbb{D} \backslash\{0\}$ then we already know that $f \in z \operatorname{Aut}(\mathbb{D})$ by Theorem 1.1(b). Assume now that $f \in z \operatorname{Aut}(\mathbb{D})$. Then $f$ is either a rotation $k z^{2}(|k|=1)$ or a function of the form $k z \varphi_{a}(z)$ with $0<|a|<1=|k|$. Without loss of generality we can suppose $k=1$ because $\rho(k z, k w)=\rho(z, w)$ for all $z$, $w \in \mathbb{D}$ if $|k|=1$. If $f(z)=z^{2}$ then (4) holds on the unit disk by Theorem 1.1(c). If $f(z)=z \varphi_{\mathrm{a}}(z)$ with $a \neq 0$ then a direct computation gives

$$
f^{[1]}(z)=\frac{1-|z|^{2}}{1-|z(a-z) /(1-\bar{a} z)|^{2}} \cdot \frac{a-2 z+\bar{a} z^{2}}{(1-\bar{a} z)^{2}} .
$$


On the other hand, (4) means that

$$
\frac{1}{2} \log \frac{1+\left|\left(f^{[1]}(0)-f^{[1]}(z)\right) /\left(1-\overline{f^{[1]}(0)} f^{[1]}(z)\right)\right|}{1-\left|\left(f^{[1]}(0)-f^{[1]}(z)\right) /\left(1-\overline{f^{[1]}(0)} f^{[1]}(z)\right)\right|}=\log \frac{1+|z|}{1-|z|}
$$

which is equivalent to

(6)

$$
\left|\frac{a-\left(\left(1-|z|^{2}\right) /\left(1-|z(a-z / 1-\bar{a} z)|^{2}\right)\right) \cdot\left(\left(a-2 z+\bar{a} z^{2}\right) /(1-\bar{a} z)^{2}\right)}{\left(1-\bar{a}\left(1-|z|^{2}\right) /\left(1-|z(a-z / 1-\bar{a} z)|^{2}\right) \cdot\left(\left(a-2 z+\bar{a} z^{2}\right) /(1-\bar{a} z)^{2}\right)\right.}\right|=\frac{2|z|}{1+|z|^{2}}
$$

due to (5) and to the fact that $\left.\psi(|z|)^{2}\right)=\psi\left(2|z| / 1+|z|^{2}\right)$ where $\psi$ is the function $\psi(t)=(1+t / 1-t)$, which is one-to-one on $(0,1)$. The left-hand side of $(6)$ can be written (after some minutes of heavy and careful calculations) as

$$
\begin{aligned}
\mid \frac{a\left(|1-\bar{a} z|^{2}-|z(a-z)|^{2}\right)(1-\bar{a} z)-}{\left(|1-\bar{a} z|^{2}-|z(a-z)|^{2}\right)(1-\bar{a} z)-}-\bar{a}\left(1-|z|^{2}\right)(1-a \bar{z})\left(a-2 z+\bar{a} z^{2}\right) \\
=\left|\frac{\left(1-|a|^{2}\right)\left(1-|z|^{2}\right)\left(2 z-a|z|^{2}-\bar{a} z^{2}\right)}{\left(1-|a|^{2}\right)\left(1-|z|^{2}\right)\left(-a \bar{z}-\bar{a} z|z|^{2}+1+|z|^{2}\right)}\right| .
\end{aligned}
$$

Therefore, after squaring, (6) is equivalent to

$$
\begin{aligned}
& \left(2 z-a|z|^{2}-\bar{a} z^{2}\right)\left(2 \bar{z}-\bar{a}|z|^{2}-a \bar{z}^{2}\right)\left(1+|z|^{2}\right)^{2} \\
& =\left(-a \bar{z}-\bar{a} z|z|^{2}+1+|z|^{2}\right)\left(-\bar{a} z-a \bar{z}|z|^{2}+1+|z|^{2}\right) 4|z|^{2} .
\end{aligned}
$$

More simplifications lead us to the equivalence of (6) and

$$
-2|a|^{2}|z|^{4}\left(|z|^{4}-2|z|^{2}+1\right)+\left(\bar{a}^{2} z^{2}+a^{2} \bar{z}^{2}\right)\left(|z|^{6}-2|z|^{4}+|z|^{2}\right)=0,
$$

or, what is the same,

$$
|z|^{2}\left(1-|z|^{2}\right)^{2}\left[-2|a|^{2}|z|^{2}+\bar{a}^{2} z^{2}+a^{2} \bar{z}^{2}\right]=0 .
$$

If $z \neq 0$ (otherwise, (6) is trivial) the last equality is the same as

$$
-2 a \bar{a} z \bar{z}+(\bar{a} z)^{2}+(a \bar{z})^{2}=0,
$$

that is, $(\bar{a} z-a \bar{z})^{2}=0$, or, equivalently, $\bar{a} z=a \bar{z}$. In other words, (6) holds if and only if $\bar{a} z \in \mathbb{R}$, which in turn means that $z \in a \mathbb{R}$, that is, (6) holds if and only if $z$ belongs to the diameter $\mathbb{D} \cap a \mathbb{R}$ passing through $a$.

With this we have proved (b) and the fact that $f$ is a 2-rotation if and only if (4) holds on all of $\mathbb{D}$. The remaining part of (c) is easy, for if (4) holds for two nonzero points lying in two distinct diameters of $\mathbb{D}$ then $f$ must be in $z$ Aut(D) but it cannot be of the form $k z \varphi_{a}(z)$ with $a \neq 0$. Consequently, $f$ is a 2-rotation and the theorem is proved. 


\section{REFERENCES}

[1] A.F. Beardon, 'The Schwarz-Pick Lemma for derivatives', Proc. Amer. Math. Soc. 125 (1997), 3255-3256.

[2] J. Dieudonné, 'Recherches sur quelques problèmes relatifs aux polynômes et aux fonctions bornées d'une variable complexe', Ann. Sci. Ecole Norm. Sup. 48 (1931), 247-358.

[3] W.F. Donoghe, Jr., Distributions and Fourier transforms (Academic Press, New York, 1966).

Departamento de Análisis Matemático Facultad de Matemáticas, Apdo. 1160

Avenida Reina Mercedes

41080 Sevilla

Spain

e-mail: lbernal@us.es
Departamento de Análisis Matemático Facultad de Matemáticas

Apdo. 1160

Avenida Reina Mercedes

41080 Sevilla

Spain

e-mail: mccm@us.es 